\title{
Multi-Layer Ionospheric VTEC Estimation for GNSS Positioning*
}

\author{
Masaharu Ohashi $^{\dagger}$, Taisuke Hattori ${ }^{\dagger}$, Yukihiro $\mathrm{Kubo}^{\dagger}$ and Sueo Sugimoto ${ }^{\dagger}$
}

\begin{abstract}
In this paper, the multi-layer ionospheric model based regional VTEC (Vertical Total Electron Content) estimation methods applied by GNSS (Global Navigation Satellite System) Regressive models (abbreviated as GR models) are presented. In the methods, the SCHA (Spherical Cap Harmonic Analysis) as well as the SHF (Spherical Harmonic Functions) are also applied to model the regional VTEC distributions. The ionospheric delays (or advances) over Japan are estimated by using GEONET (Gps Earth Observation NETwork) data provided from the GSI (Geospatial Information Authority of Japan). By applying the ionospheric correction based on the multi-layer model, the positioning accuracy can be improved, and consequently the regional ionosphere can be modeled more accurately by the multi-layer model.
\end{abstract}

\section{Introduction}

We have already developed the very powerful models of the observables of $L 1$ and $L 2$ carrier phases and pseudoranges based on $\mathrm{C} / \mathrm{A}$ and $\mathrm{P}(\mathrm{Y})$ codes in GPS, called as GR(GNSS Regressive) models, such that we have developed the PPP (Precise Point Positioning) algorithms[1-3], the VPPP (Very Precise Point Positioning) algorithms with multiple antennas[4] and the unified method for point as well as relative positioning $[5,6]$. Our PPP algorithms achieve the positioning accuracy in decimeter level and do not require the real-time transmitted information. Therefore our PPP algorithms are easily implemented without any external online received data[1-3].

By using the GR models for GNSS observables in the case of known positions (in the reference stations) $[5,6]$, we have derived a method to estimate the ionospheric delays (or advances) as well as the socalled integer ambiguities contained in $L 1$ and $L 2$ band carrier phase data at the reference stations. Further, we have developed the method of estimating the local models with power series for VTEC at the IPPs (Ionospheric Pierce Points) in the ionospheric singlelayer model by using GPS receivers' data at many reference stations $[7,8]$. The best numbers of degree in the series expansions for local models are examined by $\mathrm{AIC}[9]$ and $\mathrm{BIC}[10]$. Also, instead of SHF, we have applied SCHA for deriving the corresponding local (regional) ionospheric VTEC models over

\footnotetext{
* Manuscript Received Date: June 4, 2012

$\dagger$ Faculty of Science and Engineering, Ritsumeikan University; Noji-Higashi, Kusatsu city, Shiga 525-8577, JAPAN

Key Words: ionosphere, TEC model, spherical cap harmonic analysis, GNSS, GPS.
}

Japan using GEONET data[11].

In this paper, our previous works described above are extended such that the ionosphere is modeled by the sum of more than one layer, i.e. multi-layer model instead of single-layer model. Accuracy of the present local models of the VTEC over Japan are examined through the SPP(Standard Point Positioning) experiments using GEONET data. In the experiments, the SPP results of using the GIM (Global Ionosphere Maps) provided by the IGS (International GNSS Service), and our models based on GR models with SCHA applied by single and multi-layer ionospheric models are compared. Finally we discuss which model is better for expressing the regional VTEC over Japan.

\section{GNSS Regression Model}

\subsection{Fundamental Equations}

In general, two types of measurements called as code pseudorange and carrier phase are obtained by GPS receiver. The pseudorange $\rho_{k}^{p}$ is a measurement of the distance between the satellite $p$ and the receiver $k$. The pseudorange $\rho_{k}^{p}$ is measured by multiplying the time difference between signal reception $t_{k}$ and signal transmission $t^{p}$, and the nominal speed of light in a vacuum $c\left(\approx 2.99792458 \times 10^{8}[\mathrm{~m} / \mathrm{s}]\right)$ as follows,

$$
\begin{aligned}
\rho_{k}^{p} & =c \cdot\left(t_{k}-t^{p}\right)+e_{k}^{p} \\
& =r_{k}^{p}+e_{k}^{p}
\end{aligned}
$$

where $e_{k}^{p}$ denote measurement error. Also, $r_{k}^{p}$ is the geometric distance between the receiver $k$ and the satellite $p$. Namely,

$$
r_{k}^{p}=\sqrt{\left(x_{k}-x^{p}\right)^{2}+\left(y_{k}-y^{p}\right)^{2}+\left(z_{k}-z^{p}\right)^{2}}
$$


where $k \equiv\left[x_{k}, y_{k}, z_{k}\right]^{\mathrm{T}}$ and $s^{p} \equiv\left[x^{p}, y^{p}, z^{p}\right]^{\mathrm{T}}$ are the known receiver position and the $p$-th satellite position, respectively. The carrier phase $\varphi_{k}^{p}$ is the difference between the phase $\varphi_{k}$ of the signal generated by the receiver oscillator at the signal reception time and the phase $\varphi^{p}$ of the signal generated by the satellite at the transmission time. Since only the instantaneous fractional phase can be measured when the receiver initially starts to track the signal, the integer number $N_{k}^{p}$ of complete cycles is unknown. $N_{k}^{p}$ is called the carrier phase integer ambiguity. From the above, the carrier phase $\varphi_{k}^{p}$ is modeled as follows,

$$
\begin{aligned}
\varphi_{k}^{p} & =\varphi_{k}-\varphi^{p}+N_{k}^{p}+\varepsilon_{k}^{p} \\
& =f \cdot\left(t_{k}-t^{p}\right)+N_{k}^{p}+\varepsilon_{k}^{p} \\
& =\frac{1}{\lambda} r_{k}^{p}+N_{k}^{p}+\varepsilon_{k}^{p}
\end{aligned}
$$

where $\varepsilon_{k}^{p}$ denote measurement error and $f$ and $\lambda$ are the frequency and the wave length of the carrier wave.

In addition, measurements contain errors such as clock error, hardware bias and atomospheric error. Therefore, we consider the following fundamental measurements of the pseudorange $\rho_{C A, k}^{p}, \rho_{P Y, k}^{p}$ based on $\mathrm{C} / \mathrm{A}, \mathrm{P}(\mathrm{Y})$ codes, and $L 1$ and $L 2$ band carrier phases $\Phi_{L 1, k}^{p}, \Phi_{L 2, k}^{p}$, respectively, for the known position $k$ and satellite $p$ as follows

$$
\begin{aligned}
\rho_{C A, k}^{p}= & r_{k}^{p}+c\left(\delta t_{k}-\delta t^{p}\right)+\delta I_{k}^{p}+\delta T_{k}^{p} \\
& +\delta b_{C A, k}+\delta b_{C A}^{p}+e_{C A, k}^{p} \\
\rho_{P Y, k}^{p}= & r_{k}^{p}+c\left(\delta t_{k}-\delta t^{p}\right)+\frac{f_{1}^{2}}{f_{2}^{2}} \delta I_{k}^{p}+\delta T_{k}^{p} \\
& +\delta b_{P Y, k}+\delta b_{P Y}^{p}+e_{P Y, k}^{p} \\
\Phi_{L 1, k}^{p}= & \lambda_{1} \varphi_{L 1, k}^{p} \\
= & r_{k}^{p}+c\left(\delta t_{k}-\delta t^{p}\right)-\delta I_{k}^{p}+\delta T_{k}^{p}+\lambda_{1} N_{L 1, k}^{p} \\
& +\delta b_{L 1, k}+\delta b_{L 1}^{p}+\lambda_{1} \varepsilon_{L 1, k}^{p} \\
\Phi_{L 2, k}^{p}= & \lambda_{2} \varphi_{L 2, k}^{p} \\
= & r_{k}^{p}+c\left(\delta t_{k}-\delta t^{p}\right)-\frac{f_{1}{ }^{2}}{f_{2}{ }^{2}} \delta I_{k}^{p}+\delta T_{k}^{p}+\lambda_{2} N_{L 2, k}^{p} \\
& +\delta b_{L 2, k}+\delta b_{L 2}^{p}+\lambda_{2} \varepsilon_{L 2, k}^{p}
\end{aligned}
$$

where $f_{i}$ and $\lambda_{i}$ are the frequency and the wave length of the $L i$ carrier wave, $f_{1}=1575.42[\mathrm{MHz}], f_{2}=1227.60$ [MHz]. In (4)-(7), the so-called receiver's hardware biases, $\left\{\delta b_{C A, k}, \delta b_{P Y, k}, \delta b_{L 1, k}, \delta b_{L 2, k}\right\}$, and the satellite's hardware biases, $\left\{\delta b_{C A}^{s^{p}}, \delta b_{P Y}^{s^{p}}, \delta b_{L 1}^{s^{p}}, \delta b_{L 2}^{s^{p}}\right\}$, are contained in the observed data. Further in (4)-(7), $\delta I_{k}^{p}, \delta T_{k}^{p}$ reflect the delay or the advance associated with the propagation of the $L 1$ signal through the ionosphere and the troposphere, respectively. $\delta t_{k}, \delta t^{p}$ are the clock errors of the receiver $k$ and the satellite $p . \quad N_{L i, k}^{p}$ denotes the phase ambiguity between the satellite $p$ and the receiver $k$ in $L i$ band, and $e, \varepsilon$ denote measurement errors of code pseudorange and carrier phase.

When $n_{s}$ satellites are visible at receiver $k$, the measurements of (4)-(7) can be obtained from each satellite $p=1, \cdots, n_{s}$. Thus totaly $4 n_{s}$ measurements are obtained, and they can be expressed in a vector- matrix from as follows,

$$
\boldsymbol{y}_{k}=H_{k} \boldsymbol{\theta}_{k}+\boldsymbol{v}_{k}
$$

where

$$
\begin{aligned}
& \boldsymbol{y}_{k} \in \mathbb{R}^{4 n_{s}}, \quad H_{k} \in \mathbb{R}^{4 n_{s} \times\left(10 n_{s}+5\right)}, \\
& \boldsymbol{\theta}_{k} \in \mathbb{R}^{10 n_{s}+5}, \quad \boldsymbol{v}_{k} \in \mathbb{R}^{4 n_{s}},
\end{aligned}
$$

and

$$
\begin{aligned}
& \boldsymbol{y}_{k} \equiv\left[\boldsymbol{\rho}_{C A, k}^{\mathrm{T}}, \boldsymbol{\rho}_{P Y, k}^{\mathrm{T}}, \boldsymbol{\Phi}_{L 1, k}^{\mathrm{T}}, \boldsymbol{\Phi}_{L 2, k}^{\mathrm{T}}\right]^{\mathrm{T}}
\end{aligned}
$$

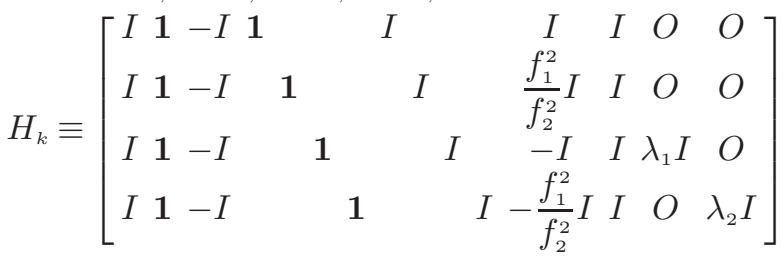

$$
\begin{aligned}
& \boldsymbol{\theta}_{k} \equiv\left[\boldsymbol{r}_{k}^{\mathrm{T}}, c \delta t_{k}, \boldsymbol{c} \boldsymbol{\delta} \boldsymbol{t}^{s \mathrm{~T}}, \boldsymbol{\delta} \boldsymbol{b}_{k}^{\mathrm{T}}, \boldsymbol{\delta} \boldsymbol{b}^{s \mathrm{~T}}\right. \text {, } \\
& \left.\boldsymbol{\delta} \boldsymbol{I}_{k}^{\mathrm{T}}, \boldsymbol{\delta} \boldsymbol{T}_{k}^{\mathrm{T}}, \boldsymbol{N}_{L 1, k}^{\mathrm{T}}, \boldsymbol{N}_{L 2, k}^{\mathrm{T}}\right]^{\mathrm{T}} \\
& \boldsymbol{\delta} \boldsymbol{b}_{k} \equiv\left[\delta b_{C A, k}, \delta b_{P Y, k}, \delta b_{L 1, k}, \delta b_{L 2, k}\right]^{\mathrm{T}} \\
& \boldsymbol{\delta} \boldsymbol{b}^{s} \equiv\left[\boldsymbol{\delta} \boldsymbol{b}_{C A}^{s}{ }^{\mathrm{T}}, \boldsymbol{\delta} \boldsymbol{b}_{P Y}^{s}{ }^{\mathrm{T}}, \boldsymbol{\delta} \boldsymbol{b}_{L 1}^{s}{ }^{\mathrm{T}}, \boldsymbol{\delta} \boldsymbol{b}_{L 2}^{s}{ }^{s}\right]^{\mathrm{T}} \\
& \boldsymbol{v}_{k} \equiv\left[\boldsymbol{e}_{C A, k}^{\mathrm{T}}, \boldsymbol{e}_{P Y, k}^{\mathrm{T}}, \boldsymbol{\varepsilon}_{L 1, k}^{\mathrm{T}}, \boldsymbol{\varepsilon}_{L 2, k}^{\mathrm{T}}\right]^{\mathrm{T}}
\end{aligned}
$$

and $\mathbf{1} \equiv[1, \cdots, 1]^{\mathrm{T}}, n_{s} \times 1$ vector, $I$ denote the $n_{s} \times n_{s}$ unit matrix. The measurements $\boldsymbol{\rho}_{C A, k}, \boldsymbol{\rho}_{P Y, k}, \boldsymbol{\Phi}_{L 1, k}$, $\boldsymbol{\Phi}_{L 2, k}$ and the unknown variables $\boldsymbol{r}_{k}, \boldsymbol{c} \boldsymbol{\delta} \boldsymbol{t}^{s}, \boldsymbol{\delta} \boldsymbol{b}_{C A}^{s}, \boldsymbol{\delta} \boldsymbol{b}_{P Y}^{s}$, $\boldsymbol{\delta} \boldsymbol{b}_{L 1}^{s}, \boldsymbol{\delta} \boldsymbol{b}_{L 2}^{s}, \boldsymbol{\delta} \boldsymbol{I}_{k}, \boldsymbol{\delta} \boldsymbol{T}_{k}, \boldsymbol{N}_{L 1, k}, \boldsymbol{N}_{L 2, k}$ are $n_{s} \times 1$ vectors, for example,

$$
\boldsymbol{\rho}_{C A, k} \equiv\left[\rho_{C A, k}^{1}, \cdots, \rho_{C A, k}^{n_{s}}\right]^{\mathrm{T}} .
$$

Similarly, measurement errors $\boldsymbol{e}_{C A, k}, \boldsymbol{e}_{P Y, k}, \boldsymbol{\varepsilon}_{L 1, k}, \boldsymbol{\varepsilon}_{L 2, k}$ are $n_{s} \times 1$ vectors, for example,

$$
\boldsymbol{e}_{C A, k} \equiv\left[e_{C A, k}^{1}, \cdots, e_{C A, k}^{n_{s}}\right]^{\mathrm{T}} .
$$

In contrast, receiver's clock error and hardware biases $c \delta t_{k}, \delta b_{C A, k}, \delta b_{P Y, k}, \delta b_{L 1, k}, \delta b_{L 2, k}$ have no relation to number of satellites, i.e. scalar quantities.

\subsection{Preprocessing on Measurement Data}

In this paper, the ionospheric term $\delta I_{k}$ in (8) is focused on, and the ionospheric models are derived in the following sections. For this purpose, the unknown parameters in (8) can be greatly reduced by taking so-called the geometry-free combination[12]. By subtracting (4) from (5), the geometric distance $r_{k}^{p}$, clock errors $\delta t_{k}, \delta t^{p}$ and tropospheric delay $\delta T_{k}^{p}$ can be eliminated as follows,

$$
\begin{aligned}
\rho_{P Y, k}^{p}-\rho_{C A, k}^{p}= & \left(1-f_{1}^{2} / f_{2}^{2}\right) \delta I_{k}^{p}+\left(\delta b_{P Y, k}-\delta b_{C A, k}\right) \\
& +\left(\delta b_{P Y}^{p}-\delta b_{C A}^{p}\right)+\left(e_{P Y, k}^{p}-e_{C A, k}^{p}\right) .
\end{aligned}
$$

This operation is called code-code geometry-free combination. Similarly, by subtracting (6) from (7), the phase-phase geometry-free combination can be formed. Therefore, the geometry-free operation on (8) can be expressed as follows,

$$
D \boldsymbol{y}_{k}=D H_{k} \boldsymbol{\theta}_{k}+D \boldsymbol{v}_{k}
$$


where

$$
D \equiv\left[\begin{array}{cccc}
-I & I & O & O \\
O & O & -I & I
\end{array}\right]
$$

Now, define

$$
\boldsymbol{y}_{d, k} \equiv D \boldsymbol{y}_{k}, \quad H_{d, k} \equiv D H_{k}, \quad \boldsymbol{v}_{d, k} \equiv D \boldsymbol{v}_{k},
$$

then (8) is reduced to

$$
\boldsymbol{y}_{d, k}=H_{d, k} \boldsymbol{\theta}_{d, k}+\boldsymbol{v}_{d, k}
$$

where

$$
\begin{array}{ll}
\boldsymbol{y}_{d, k} \in \mathbb{R}^{2 n_{s}}, & H_{d, k} \in \mathbb{R}^{2 n_{s} \times 3 n_{s}}, \\
\boldsymbol{\theta}_{d, k} \in \mathbb{R}^{3 n_{s}}, & \boldsymbol{v}_{d, k} \in \mathbb{R}^{2 n_{s}},
\end{array}
$$

and

$$
\begin{gathered}
\boldsymbol{y}_{d, k} \equiv\left[\begin{array}{c}
\boldsymbol{\rho}_{P Y, k}-\boldsymbol{\rho}_{C A, k} \\
\boldsymbol{\Phi}_{L 2, k}-\boldsymbol{\Phi}_{L 1, k}
\end{array}\right], H_{d, k} \equiv\left[\begin{array}{ccc}
\left(\frac{f_{1}^{2}}{f_{2}^{2}}-1\right) I & I & O \\
-\left(\frac{f_{1}^{2}}{f_{2}^{2}}-1\right) I & O & I
\end{array}\right] \\
\boldsymbol{\theta}_{d, k} \equiv\left[\begin{array}{c}
\boldsymbol{\delta} I_{k} \\
\boldsymbol{B}_{C A-P Y, k} \\
\boldsymbol{B}_{L 1-L 2, k}
\end{array}\right], \boldsymbol{v}_{d, k} \equiv\left[\begin{array}{c}
\boldsymbol{e}_{P Y, k}-\boldsymbol{e}_{C A, k} \\
\boldsymbol{\varepsilon}_{L 2, k}-\boldsymbol{\varepsilon}_{L 1, k}
\end{array}\right] \\
\boldsymbol{B}_{C A-P Y, k} \equiv \mathbf{1}\left(\delta b_{P Y, k}-\delta b_{C A, k}\right)+\boldsymbol{\delta} \boldsymbol{b}_{P Y}^{s}-\boldsymbol{\delta} \boldsymbol{b}_{C A}^{s} \\
\boldsymbol{B}_{L 1-L 2, k} \equiv \mathbf{1}\left(\delta b_{L 2, k}-\delta b_{L 1, k}\right)+\boldsymbol{\delta} \boldsymbol{b}_{L 2}^{s}-\boldsymbol{\delta} \boldsymbol{b}_{L 1}^{s} \\
+\lambda_{2} \boldsymbol{N}_{L 2, k}-\lambda_{1} \boldsymbol{N}_{L 1, k} .
\end{gathered}
$$

By the transformations, geometric distance $r$, tropospheric delay $\delta T$, the clock errors of the receiver and the satellite $\delta t_{k}, \delta t^{p}$ are eliminated.

\section{Single-Layer Model}

Next we develop an estimation method of SCHA models of the local VTEC map in the sky over Japan. We here apply the single-layer model shown in Fig. 1. In this model, the ionosphere is modeled by a thin shell. Because GPS basically provides measurements of the ionospheric delay(or advance) between the satellite and the receiver in meter due to the slant TEC instead of VTEC, it is important to formulate the

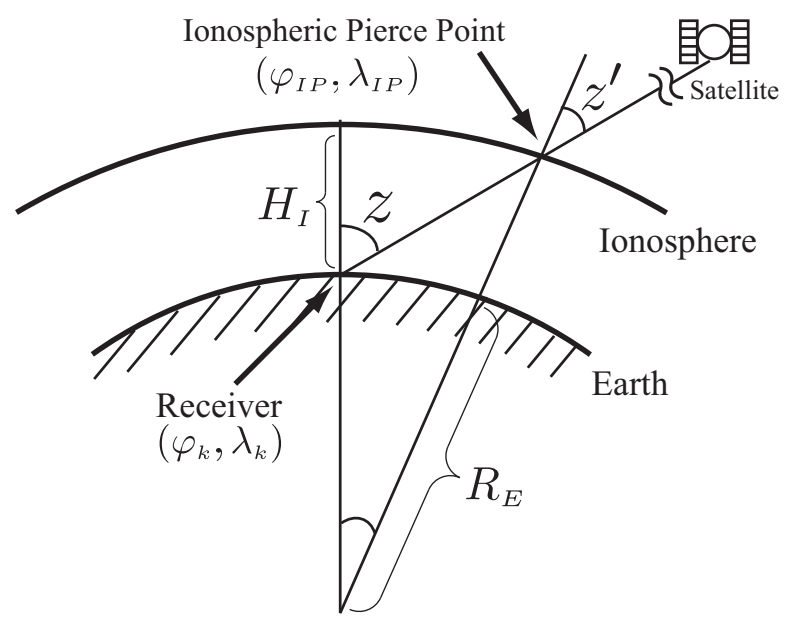

Fig. 1 Single-Layer Model relationship between the measured ionospheric delay and VTEC. Therefore, now, an elevation-dependent mapping function $M_{I}$ which transforms VTEC to the slant TEC at the IPP as follows

$$
M_{I}=\frac{1}{\cos z^{\prime}}
$$

where $z^{\prime}$ is the satellite's zenith angle at the IPP. The relationship between $z$ and $z^{\prime}$ is expressed by

$$
z^{\prime}=\arcsin \left(\frac{R_{E}}{R_{E}+H_{I}} \sin z\right)
$$

where $R_{E}(\approx 6371[\mathrm{~km}])$ is the mean earth radius and $H_{I}$ is the height of single-layer. Then we have the relationship between $\delta I$ and VTEC as follows[13]

$$
\delta I=\frac{40.3 \times 10^{16}}{f^{2}} \times M_{I} \times \mathrm{VTEC} .
$$

\subsection{Spherical Cap Harmonics Analysis (SCHA) Model}

In order to model the distribution of the VTEC, several methods such as power series expansion, SCHA have been proposed. SCHA is the harmonic function around a part of sphere and the local VTEC such as in the sky over Japan is focused on in this paper. Therefore, also from results of previous works[8,11], it can be considered the ionospheric VTEC is well modeled by SCHA. By using SCHA, the ionospheric VTEC is expressed as follows[11]

$$
\begin{aligned}
\mathrm{VTEC} & =\underset{n=0}{E\left(\beta_{I P}, s_{I P}\right)} \\
& =\sum_{m=0}^{n_{\max }} \sum_{\substack{n \\
P_{n_{l}(m)}^{m}}}^{n}\left\{\left(a_{n m} \cos m s_{I P}+b_{n m} \sin m s_{I P}\right)\right\}
\end{aligned}
$$

The parameter $\beta_{I P}$ and $s_{I P}$ are latitude and longitude at the IPP in the sun-fixed coordinate system, $a_{n m}$ and $b_{n m}$ are unknown coefficients of SCHA, the parameters $n$ and $m$ are generally referred to as the degree and order, $n_{\max }$ denotes the maximum degree of SCHA, $P_{n_{l}(m)}^{m}$ is the associated Legendre function in SCHA. Combining (12) and (13), we have the relationship

$$
\begin{aligned}
& \delta I_{k}^{p}=\frac{40.3 \times 10^{16}}{f_{1}^{2}} \times\left[\frac{1}{\cos \left(z_{k}^{p}\right)^{\prime}} \times \sum_{n=0}^{n_{\max }} \sum_{m=0}^{n}\right. \\
&\left.\left\{a_{n m} \cos m s_{I P}+b_{n m} \sin m s_{I P}\right\} P_{n_{l(m)}}^{m}\left(\sin \beta_{I P}\right)\right] .
\end{aligned}
$$

By using (15), we can formulate the ionospheric delay $\delta I_{k}$ for satellites $p=1, \cdots, n_{s}$ and receiver $k$ as follows

$$
\boldsymbol{\delta} \boldsymbol{I}_{k}=\left[\begin{array}{c}
\delta I_{k}^{1} \\
\vdots \\
\delta I_{k}^{n_{s}}
\end{array}\right]=\left[\begin{array}{ll}
A_{a, k} & A_{b, k}
\end{array}\right]\left[\begin{array}{c}
\boldsymbol{\theta}_{a} \\
\boldsymbol{\theta}_{b}
\end{array}\right] \equiv A_{k} \boldsymbol{\theta}_{c}
$$

where 


$$
A_{k} \in \mathbb{R}^{n_{s} \times\left(n_{\max }+1\right)^{2}}, \quad \boldsymbol{\theta}_{c} \in \mathbb{R}^{\left(n_{\max }+1\right)^{2}},
$$

and

$$
\begin{aligned}
& A_{a, k} \equiv\left[\begin{array}{cccccc}
A_{00, k}^{1} & \cdots & A_{n_{\max } 0, k}^{1} & A_{n_{\max 1, k}}^{1} & \cdots & A_{n_{\max } m, k}^{1} \\
\vdots & & \vdots & \vdots & & \vdots \\
A_{00, k}^{n_{s}} & \cdots & A_{n_{\max } 0, k}^{n_{s}} & A_{n_{\max } n_{s}}^{n_{s}} & \cdots & A_{n_{\max } m, k}^{n_{s}}
\end{array}\right] \\
& A_{b, k} \equiv\left[\begin{array}{cccccc}
B_{00, k}^{1} & \cdots & B_{n_{\max 0, k}}^{1} & B_{n_{\max 1, k}}^{1} \cdots & B_{n_{\max m, k}}^{1} \\
\vdots & & \vdots & \vdots & & \vdots \\
B_{00, k}^{n_{s}} & \cdots & B_{n_{\max 0, k} n_{s}}^{n_{\max }} & B_{n_{\max 1, k}}^{n_{s}} \cdots & B_{n_{\max m, k}}^{n_{s}}
\end{array}\right] \\
& A_{n m, k}^{p}=\frac{40.3 \times 10^{16}}{f_{1}^{2}} \times \frac{1}{\cos \left(z_{k}^{p}\right)^{\prime}} \\
& \times\left(\cos m s_{I P}\right) P_{n_{l(m)}}^{m}\left(\sin \beta_{I P}\right) \\
& B_{n m, k}^{p}=\frac{40.3 \times 10^{16}}{f_{1}^{2}} \times \frac{1}{\cos \left(z_{k}^{p}\right)^{\prime}} \\
& \times\left(\sin m s_{I P}\right) P_{n_{l(m)}}^{m}\left(\sin \beta_{I P}\right) \\
& \boldsymbol{\theta}_{a} \equiv\left[\begin{array}{lllll}
a_{00}|\cdots| a_{n_{\max 0} 0} & a_{n_{\max 1}} & \cdots & a_{n_{\max } m}
\end{array}\right]^{\mathrm{T}} \\
& \boldsymbol{\theta}_{b} \equiv\left[b_{00}|\cdots| b_{n_{\max 0}} b_{n_{\max 1}} \cdots b_{n_{\max m}}\right]^{\mathrm{T}} \text {. }
\end{aligned}
$$

\subsection{Measurement Equation with Single- Layer Model}

By substituting $A_{k} \boldsymbol{\theta}_{c}$ of (16) into $\boldsymbol{\delta} I_{k}$ of (10), the measurement equation is obtained as follows,

$$
\boldsymbol{y}_{d, k}=H_{d, k} \boldsymbol{\theta}_{d, k}+\boldsymbol{v}_{d, k}
$$

where

$$
\begin{aligned}
& \boldsymbol{y}_{d, k} \in \mathbb{R}^{2 n_{s}}, \quad H_{d, k} \in \mathbb{R}^{2 n_{s} \times\left(\left(n_{\max }+1\right)^{2}+2 n_{s}\right)}, \\
& \boldsymbol{\theta}_{d, k} \in \mathbb{R}^{\left(n_{\max }+1\right)^{2}+2 n_{s}}, \quad \boldsymbol{v}_{d, k} \in \mathbb{R}^{2 n_{s}},
\end{aligned}
$$

and the matrix $H_{d, k}$ and the vector $\boldsymbol{\theta}_{d, k}$ are redefined as follows,

$$
\begin{aligned}
& H_{d, k} \equiv\left[A_{k, D} \mid \begin{array}{ll}
I & \\
& I
\end{array}\right], \quad A_{k, D} \equiv\left[\begin{array}{c}
\left(\frac{f_{1}^{2}}{f_{2}^{2}}-1\right) I \\
-\left(\frac{f_{1}^{2}}{f_{2}^{2}}-1\right) I
\end{array}\right] A_{k}, \\
& \boldsymbol{\theta}_{d, k} \equiv\left[\begin{array}{c}
\boldsymbol{\theta}_{c} \\
\boldsymbol{B}_{P Y-C A, k} \\
\boldsymbol{B}_{L 2-L 1, k}
\end{array}\right] .
\end{aligned}
$$

In actual, the number of visible satellites depends on the position of the receiver and time. In this paper, it is assumed that the number of visible satellites at each receiver is the same. From (17), when we collect all of the receiver data from $n_{s}$ satellites and $M$ GEONET stations $1, \cdots, M$, we have

$$
\boldsymbol{Y}_{M}=H_{M} \boldsymbol{\theta}_{M}+\boldsymbol{v}_{M}
$$

where

$$
\begin{aligned}
& \boldsymbol{Y}_{M} \in \mathbb{R}^{2 n_{s} \cdot M}, \quad H_{M} \in \mathbb{R}^{2 n_{s} \cdot M \times\left(\left(n_{\max }+1\right)^{2}+2 n_{s} \cdot M\right)}, \\
& \boldsymbol{\theta}_{M} \in \mathbb{R}^{\left(n_{\max }+1\right)^{2}+2 n_{s} \cdot M}, \quad \boldsymbol{v}_{d, k} \in \mathbb{R}^{2 n_{s} \cdot M},
\end{aligned}
$$

and

$$
\begin{aligned}
\boldsymbol{Y}_{M} \equiv\left[\begin{array}{c}
\boldsymbol{y}_{d, 1} \\
\vdots \\
\boldsymbol{y}_{d, M}
\end{array}\right], \quad H_{M} \equiv\left[\begin{array}{ccc}
A_{1, D} & I_{2 n_{s}} & \\
\vdots & & \ddots \\
\boldsymbol{\theta}_{c} \\
A_{M, D} & & I_{2 n_{s}}
\end{array}\right], \\
\boldsymbol{\theta}_{M} \equiv\left[\begin{array}{c}
\boldsymbol{B}_{P Y-C A, 1} \\
\boldsymbol{B}_{L 2-L 1,1} \\
\vdots \\
\boldsymbol{B}_{P Y-C A, M} \\
\boldsymbol{B}_{L 2-L 1, M}
\end{array}\right], \quad v_{M} \equiv\left[\begin{array}{c}
v_{d, 1} \\
\vdots \\
v_{d, M}
\end{array}\right],
\end{aligned}
$$

and $I_{2 n_{s}}$ denote the $2 n_{s} \times 2 n_{s}$ unit matrix.

\subsection{Estimation Method with Single- Layer Model}

Because the measurement of (18) can be obtained at successive observation epochs, hereafter, the time " $t$ " is added to (18) as subscripts. Then, by applying the least squares method, we have the smoothing estimate $\hat{\theta}_{M, t}$ at time $t$ as follows

$$
\begin{aligned}
\hat{\boldsymbol{\theta}}_{M, t}=\left(\sum_{j=t-d}^{t+d} H_{M, j}^{\mathrm{T}}\right. & \left.R_{M, j}^{-1} H_{M, j}\right)^{-1} \\
& \times\left(\sum_{j=t-d}^{t+d} H_{M, j}^{\mathrm{T}} R_{M, j}^{-1} Y_{M, j}\right)
\end{aligned}
$$

where

$$
R_{M, j}=\operatorname{Cov}\left[\boldsymbol{v}_{M, j}\right] .
$$

\subsection{Computation of Ionospheric Delays for Positioning on Single-Layer Model}

By sending the estimated SCHA coefficients $\hat{\boldsymbol{\theta}}_{a, t}$, $\hat{\boldsymbol{\theta}}_{b, t}$ to GNSS users, they can calculate and correct the ionospheric delays from (15). In order to reduce the computational burden and data volume to be sent to users, the coefficients are typically estimated every two hours by using two hours observation data[14], i.e. $t$ and $d$ are every 2 hours and 1 hour respectively in (19). In this case, the ionospheric delay at a specific time can be computed by the time interpolation algorithm[14]. For example, by using the estimates of SCHA coefficients at time $t_{1}, t_{2}$, the ionospheric delays $\hat{\delta I_{k, t_{1}}^{p}}$ and $\hat{\delta I}_{k, t_{2}}^{p}$ can be obtained from (15). Then the ionospheric delay at time $t\left(t_{1} \leq t \leq t_{2}\right)$ can be computed by

$$
\hat{\delta I}_{k, t}^{p}=\frac{t_{2}-t}{t_{2}-t_{1}} \hat{\delta I_{k, t_{1}}^{p}}+\frac{t-t_{1}}{t_{2}-t_{1}} \hat{\delta I}_{k, t_{2}}^{p} .
$$

\section{Multi-Layer Model}

The actual ionosphere has thickness and the density of electrons varies with height. Therefore it is expected that the high accurate ionospheric model can be developed with the assumption that there are several ionospheric thin shells between the receiver and the satellite. With this consideration, in this paper, we suppose that the ionosphere consists of more than 


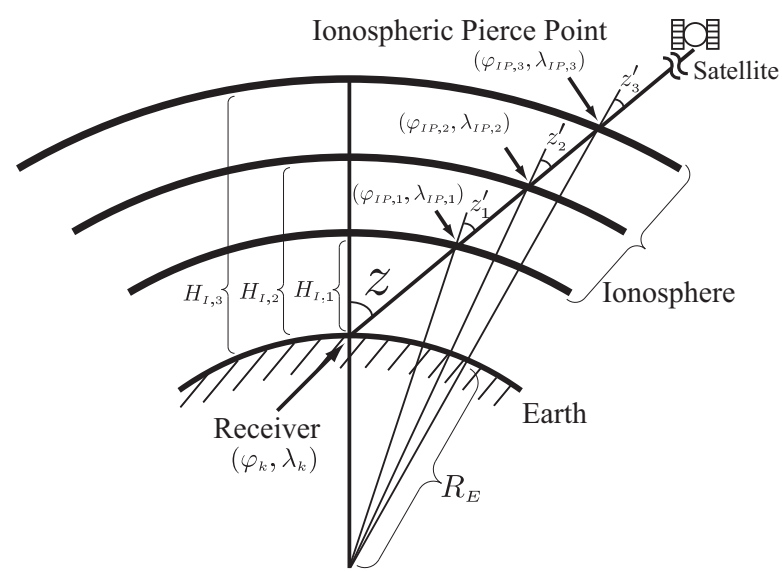

Fig. 2 Multi-Layer Model

one layer e.g. 3-layer model shown in Fig. 2. Mapping function $M_{I, i}$ of the IPP of $i$-th layer is assumed as

$$
M_{I, i}=\frac{1}{\cos z_{i}^{\prime}}
$$

where $z_{i}^{\prime}$ is the satellite's zenith angle at the IPP of $i$-th layer and the relationship between $z$ and $z_{i}^{\prime}$ is

$$
z_{i}^{\prime}=\arcsin \left(\frac{R_{E}}{R_{E}+H_{I, i}} \sin z\right)
$$

where $H_{I, i}$ shows the height of $i$-th layer. Similarly to (14) and (15), the ionospheric VTEC and delay for $i$-th layer: $\mathrm{VTEC}_{i}$ and $\delta I_{k, i}^{p}$ can be modulated by SCHA.

\subsection{Ionospheric delay on Multi-Layer Model}

Then, in this paper, we assume the (total) ionospheric delay $\delta I_{k}^{p}$ between satellite $p$ and receiver $k$ as sum of product of unknown coefficient and estimated ionospheric delays $\delta I_{k, i}^{p}(i=1, \cdots, L)$ based on the single-layer model with $L$ different heights $H_{I, i}(i=$ $1, \cdots, L)$ as follows

$$
\delta I_{k}^{p}=\sum_{i=1}^{L} \alpha_{i} \times \hat{\delta I_{k, i}^{p}}
$$

where $\alpha_{i}$ is the weighted factor to $i$-th layer. We suppose that the weighted factor is greater than or equal to 0 and less than or equal to 1 , moreover sum of the weighted factors is equal to 1 as follows,

$$
\begin{aligned}
& 0 \leq \alpha_{i} \leq 1 \\
& \sum_{i=1}^{L} \alpha_{i}=1 .
\end{aligned}
$$

By using (24), we can formulate the ionospheric delay $\delta I_{k}$ for satellites $p=1, \cdots, n_{s}$ and receiver $k$ as follows

$$
\boldsymbol{\delta} \boldsymbol{I}_{k}=\left[\begin{array}{c}
\delta I_{k}^{1} \\
\vdots \\
\delta I_{k}^{n_{s}}
\end{array}\right]=\left[\begin{array}{ccc}
\hat{\delta I_{k, 1}^{1}} & \cdots & \hat{\delta I_{k, L}^{1}} \\
\vdots & & \vdots \\
\hat{\delta I_{k, 1}^{n_{s}}} & \cdots & \hat{\delta I_{k, L}^{n_{s}}}
\end{array}\right]\left[\begin{array}{c}
\alpha_{1} \\
\vdots \\
\alpha_{L}
\end{array}\right]=A_{\delta I, k} \boldsymbol{\theta}_{\alpha},
$$

where

$$
A_{\delta I, k} \in \mathbb{R}^{n_{s} \times L}, \quad \boldsymbol{\theta}_{\alpha} \in \mathbb{R}^{L} .
$$

\subsection{Measurement Equation with Multi- Layer Model}

In (10) with (27), we can express the following equation

$$
\boldsymbol{y}_{d, k}=H_{d, k} \boldsymbol{\theta}_{d, k}+\boldsymbol{v}_{d, k}
$$

where

$$
\begin{aligned}
& \boldsymbol{y}_{d, k} \in \mathbb{R}^{2 n_{s}}, \quad H_{d, k} \in \mathbb{R}^{2 n_{s} \times\left(L+2 n_{s}\right)}, \\
& \boldsymbol{\theta}_{d, k} \in \mathbb{R}^{\left(L+2 n_{s}\right)}, \quad \boldsymbol{v}_{d, k} \in \mathbb{R}^{2 n_{s}},
\end{aligned}
$$

and the matrix $H_{d, k}$ and the vector $\boldsymbol{\theta}_{d, k}$ are redefined as follows,

$H_{d, k} \equiv\left[A_{\delta I, k, D} \mid \begin{array}{rr}I & \\ & I\end{array}\right], \quad A_{\delta I, k, D} \equiv\left[\begin{array}{c}\left(\frac{f_{1}^{2}}{f_{2}^{2}}-1\right) I \\ -\left(\frac{f_{1}^{2}}{f_{2}^{2}}-1\right) I\end{array}\right] A_{\delta I, k}$

$\boldsymbol{\theta}_{d, k} \equiv\left[\begin{array}{c}\boldsymbol{\theta}_{\alpha} \\ \boldsymbol{B}_{C A-P Y, k} \\ \boldsymbol{B}_{L 1-L 2, k}\end{array}\right]$.

When we collect all of the receiver data from $M$ GEONET stations $1, \cdots, M$, we have

$$
\boldsymbol{Y}_{M}=H_{M} \boldsymbol{\theta}_{M}+\boldsymbol{v}_{M}
$$

where

$$
\begin{aligned}
& \boldsymbol{Y}_{M} \in \mathbb{R}^{2 n_{s} \cdot M}, \quad H_{M} \in \mathbb{R}^{2 n_{s} \cdot M \times\left(L+2 n_{s} \cdot M\right)} \\
& \boldsymbol{\theta}_{M} \in \mathbb{R}^{L+2 n_{s} \cdot M}, \quad \boldsymbol{v}_{M} \in \mathbb{R}^{2 n_{s} \cdot M},
\end{aligned}
$$

and the matrix $H_{M}$ and the vector $\boldsymbol{\theta}_{M}$ are redefined as follows,

$H_{M}=\left[\begin{array}{cccc}A_{\delta I, 1, D} & I_{2 n_{s}} & & \\ \vdots & & \ddots & \\ A_{\delta I, M, D} & & & I_{2 n_{s}}\end{array}\right], \quad \boldsymbol{\theta}_{M} \equiv\left[\begin{array}{c}\boldsymbol{\theta}_{\alpha} \\ \boldsymbol{B}_{C A-P Y, 1} \\ \boldsymbol{B}_{L 1-L 2,1} \\ \vdots \\ \boldsymbol{B}_{C A-P Y, M} \\ \boldsymbol{B}_{L 1-L 2, M}\end{array}\right]$.

\subsection{Estimation Method with Multi- Layer Model}

Now, by the same reasons in Section 3.3, the subscript "t" is introduced to (29). Then, with the constraints of (25) and (26), the estimates $\hat{\boldsymbol{\theta}}_{M, t}$ at time $t$ is obtained by using the least squares criterion as follows,

$$
\hat{\boldsymbol{\theta}}_{M, t}=\underset{\theta_{M, t}}{\arg \min }\left\|\boldsymbol{Y}_{M, t t}-H_{M, t t} \boldsymbol{\theta}_{M, t}\right\|_{R_{M, t t}^{-1}}^{2}
$$

subject to

$\boldsymbol{g}_{E}\left(\boldsymbol{\theta}_{M, t}\right)=\left[\mathbf{1}_{L}^{\mathrm{T}} \mid \mathbf{0}_{2 n_{s} \cdot M}^{\mathrm{T}}\right] \boldsymbol{\theta}_{M, t}-1=0$ 
$\boldsymbol{g}_{I}\left(\boldsymbol{\theta}_{M, t}\right)=\left[\begin{array}{c|c}-I_{L} & O_{L \times 2 n_{s} \cdot M} \\ \hline I_{L} & O_{L \times 2 n_{s} \cdot M}\end{array}\right] \boldsymbol{\theta}_{M, t}-\left[\begin{array}{c}\mathbf{0}_{L} \\ \hline-\mathbf{1}_{L}\end{array}\right] \leq\left[\begin{array}{c}\mathbf{0}_{L} \\ \hline \mathbf{0}_{L}\end{array}\right]$

where

$$
\begin{aligned}
\boldsymbol{Y}_{M, t t}= & {\left[\begin{array}{c}
\boldsymbol{Y}_{M, t-d} \\
\vdots \\
\boldsymbol{Y}_{M, t} \\
\vdots \\
\boldsymbol{Y}_{M, t+d}
\end{array}\right], \quad H_{M, t t}=\left[\begin{array}{c}
H_{M, t-d} \\
\vdots \\
H_{M, t} \\
\vdots \\
H_{M, t+d}
\end{array}\right], } \\
R_{M, t t}= & \operatorname{Cov}\left[\begin{array}{c}
\boldsymbol{v}_{M, t-d} \\
\vdots \\
\boldsymbol{v}_{M, t-d} \\
\vdots \\
\boldsymbol{v}_{M, t+d}
\end{array}\right],
\end{aligned}
$$

and $\mathbf{1}_{L} \equiv[1, \cdots, 1]^{\mathrm{T}}, L \times 1$ vector, $\mathbf{0}_{2 n_{s} \cdot M} \equiv[1, \cdots, 1]^{\mathrm{T}}$, $L \times 1$ vector, $\mathbf{0}_{L} \equiv[1, \cdots, 1]^{\mathrm{T}}, L \times 1$ vector, $I_{L}$ denote the $L \times L$ unit matrix, $O_{L \times 2 n_{s} \cdot M}$ denote the $L \times 2 n_{s} \cdot M$ zero matrix. This problem, the minimization with equality and inequality constraints, is solved by the algorithm applied by the sequential quadratic programing techniques. The details of the algorithm can be found in[15].

\subsection{Computation of Ionospheric Delays for Positioning on Multi-Layer Model}

Similarly to Section 3.4, the $i$-th layer ionospheric delay at time $t\left(t_{1} \leq t \leq t_{2}\right)$ can be computed by

$$
\hat{\delta I_{k, i, t}^{p}}=\frac{t_{2}-t}{t_{2}-t_{1}} \hat{\delta I_{k, i, t_{1}}^{p}}+\frac{t-t_{1}}{t_{2}-t_{1}} \hat{\delta I_{k, i, t_{2}}^{p}}
$$

Then, with the estimates of (30-32), the total delay between satellite $p$ and receiver $k$ at time $t$ can be computed by

$$
\hat{\delta I}_{k, t}^{p}=\sum_{i=1}^{L} \hat{\alpha}_{i, t} \times \hat{\delta I}_{k, i, t}^{p}
$$

\section{Experimental Results}

\subsection{VTEC Maps with Proposed Meth- ods}

From (19) and (30)-(32), we developed VTEC maps in the sky over Japan with the single-layer and multi-layer model. We estimated parameters on June 1st, 2010, 8:00 and 10:00 (UTC) by using GEONET data from 7:00 to 9:00 (UTC) for the parameters at 8:00 and the data from 9:00 to 11:00 (UTC) for the parameters at 10:00. Therefore, in this case, $t$ is $8: 00$ or 10:00 and $d$ is 1 hour in (19) and (30). The sampling time interval of data was 10 minutes. The total number of the used GEONET stations was 30. The 30 stations are selected such that IPPs widely spread in the sky over Japan, the number of measurements are not too large for computation. Fig. 3 shows the locations of the used stations. In the experiment, $H_{I}=350[\mathrm{~km}]$ was applied for the single-layer model. On the other hand, for the multi-layer model, $L=3$ such that $H_{I, 1}=250, H_{I, 2}=350$ and $H_{I, 3}=450[\mathrm{~km}]$ were applied. Maximum order of the SCHA was 2.

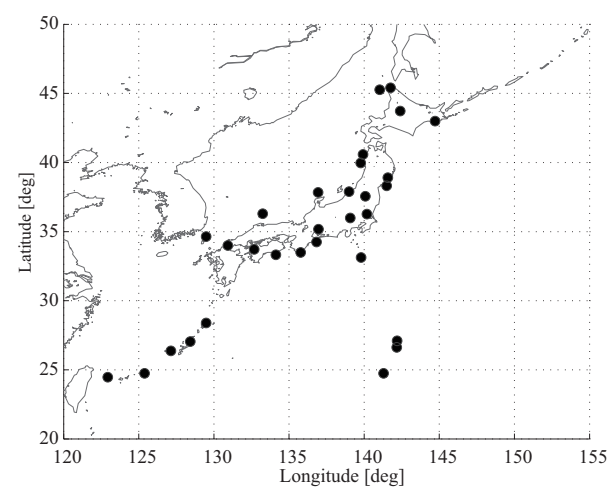

Fig. 3 GEONET stations for VTEC estimation

In Table 1, 2, the estimated SCHA's coefficients $a_{n m}, b_{n m}$ with single-layer model at 8:00 and 10:00 (UTC) are summarized. In Table 3, the estimated coefficients $\alpha$ of each layers with multi-layer model are summarized.

Fig. 4 shows VTEC map with the single-layer model at 8:00 (UTC) and Figs. 5-7 show VTEC maps with multi-layer at 8:00 (UTC) model of each height, where VTEC values are shown in units of TECU(TEC Units), defined as $10^{16}$ electrons per square meter. From Fig. 4, it can be seen that VTEC value increases from high to low latitude due to solar activity. Furthermore, VTEC of $250[\mathrm{~km}]$ high had comparatively higher value than other ones on that time. From Figs. 5-7, VTEC distribution of each layer showed the similar tendency the single-layer model.

\subsection{Comparison of Positioning Accu- racy}

The experiments of the SPP[13] with three ionospheric models were carried out by using real receiver data. The observation data were obtained from GEONET reference station (Fuji) on June 1st, 2010, 8:00-10:00 (UTC). Then ionospheric mdoels at 8:00 and 10:00 are applied to the positioning. Fuji is located Fuji city, Shizuoka prefecture, Japan. At the station, the Trimble NETRS receiver with TRM29659.00 antenna observed at every 30 seconds. In the experiments, an elevation cut-off angle of 10 [deg] was applied. The observation of the satellite positions and errors of satellite clocks were obtained from the broadcast ephemeris. Fig. 8 shows the position error with GIM. Fig. 9 shows the positioning errors with single-layer model. Also, Fig. 10 shows the positioning errors with multi-layer model.

We here use the position's true values provide by GSI (Geospatial Information Authority of Japan). 
Table 1 Estimated coefficients of SCHA at 8:00 UTC

\begin{tabular}{|r|r|r|r|}
\hline$H_{I, i}$ & $250[\mathrm{~km}]$ & $350[\mathrm{~km}]$ & $450[\mathrm{~km}]$ \\
\hline \hline$\hat{a}_{00}$ & 23.899 & 24.138 & 24.760 \\
\hline$\hat{a}_{10}$ & 12.500 & 9.924 & 8.880 \\
\hline$\hat{a}_{11}$ & 11.703 & 14.699 & 15.760 \\
\hline$\hat{a}_{20}$ & -9.823 & -7.095 & -5.634 \\
\hline$\hat{a}_{21}$ & 3.502 & 3.944 & 4.028 \\
\hline$\hat{a}_{22}$ & -32.083 & -30.614 & -30.707 \\
\hline$\hat{b}_{11}$ & -45.121 & -39.985 & -38.410 \\
\hline$\hat{b}_{21}$ & 1.898 & 1.737 & 1.159 \\
\hline$\hat{b}_{22}$ & -21.400 & -24.898 & -26.199 \\
\hline
\end{tabular}

Table 2 Estimated coefficients of SCHA at 10:00 UTC

\begin{tabular}{|c|r|r|r|}
\hline$H_{I, i}$ & \multicolumn{1}{|c|}{$250[\mathrm{~km}]$} & \multicolumn{1}{c|}{$350[\mathrm{~km}]$} & \multicolumn{1}{c|}{$450[\mathrm{~km}]$} \\
\hline \hline$\hat{a}_{00}$ & 68.103 & 67.182 & 59.988 \\
\hline$\hat{a}_{10}$ & -74.772 & -83.230 & -75.204 \\
\hline$\hat{a}_{11}$ & 159.311 & 143.007 & 128.373 \\
\hline$\hat{a}_{20}$ & 24.366 & 31.504 & 31.147 \\
\hline$\hat{a}_{21}$ & 34.356 & 23.372 & 23.097 \\
\hline$\hat{a}_{22}$ & -15.091 & -5.037 & -0.024 \\
\hline$\hat{b}_{11}$ & -16.423 & 2.885 & 12.299 \\
\hline$\hat{b}_{21}$ & 27.936 & 28.881 & 25.684 \\
\hline$\hat{b}_{22}$ & -211.518 & -188.235 & -166.975 \\
\hline
\end{tabular}

Table 3 Estimated coefficients on Multi-Layer Model

\begin{tabular}{|r|c|c|c|}
\hline UTC & $\hat{\alpha}_{1}$ & $\hat{\alpha}_{2}$ & $\hat{\alpha}_{3}$ \\
\hline \hline $8: 00$ & 0.4302 & 0.2563 & 0.3134 \\
\hline $10: 00$ & 0.3229 & 0.3229 & 0.3542 \\
\hline
\end{tabular}

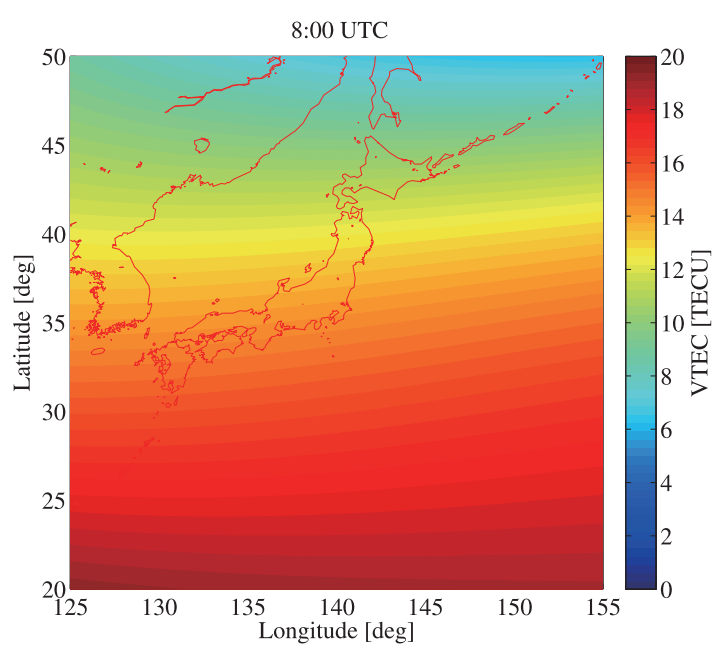

Fig. 4 VTEC map (Single-Layer model)

The statistics of positioning results are summarized in Table 1. In Table 1, the bias, standard deviation and RMS values of positioning errors are shown for East, North, and Up (Vertical) directions and horizontal plane (2D).

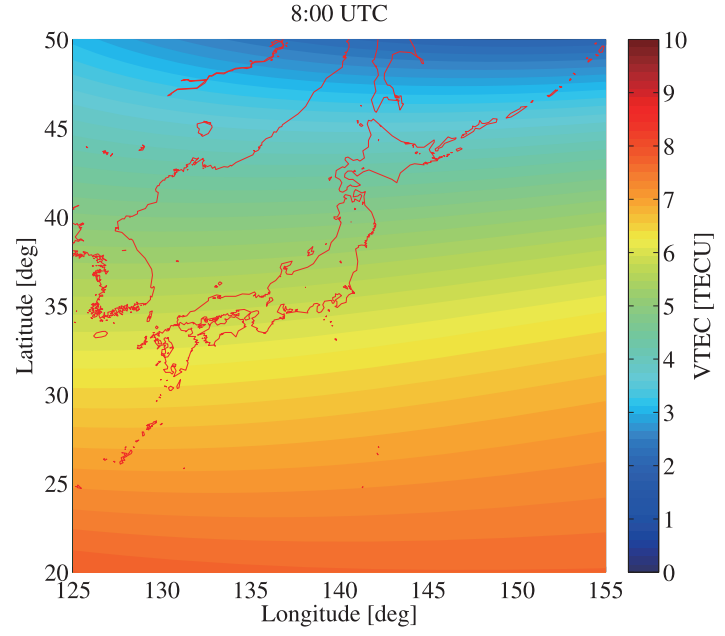

Fig. 5 VTEC map (3-Layer model) of 250[km] high

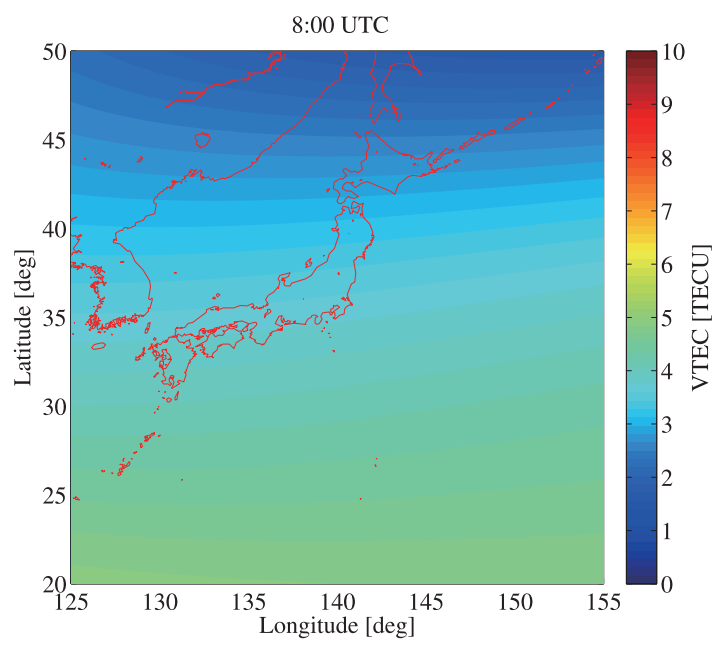

Fig. 6 VTEC map (3-Layer model) of $350[\mathrm{~km}]$ high

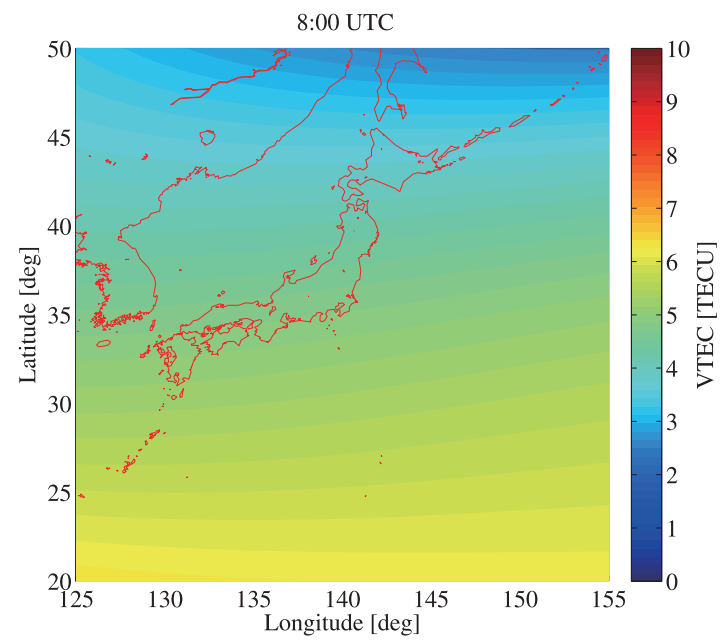

Fig. 7 VTEC map (3-Layer model) of $450[\mathrm{~km}]$ high

From Table 4, we can see that the error of 2D was improved with the proposed multi-layer method whereas the error of up direction degraded worth than with single-layer method. 


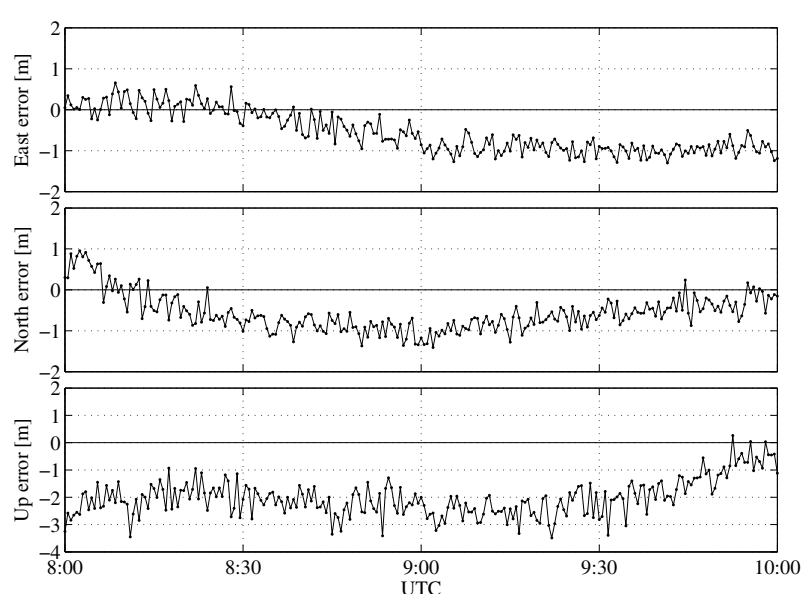

Fig. 8 Positioning errors (GIM)

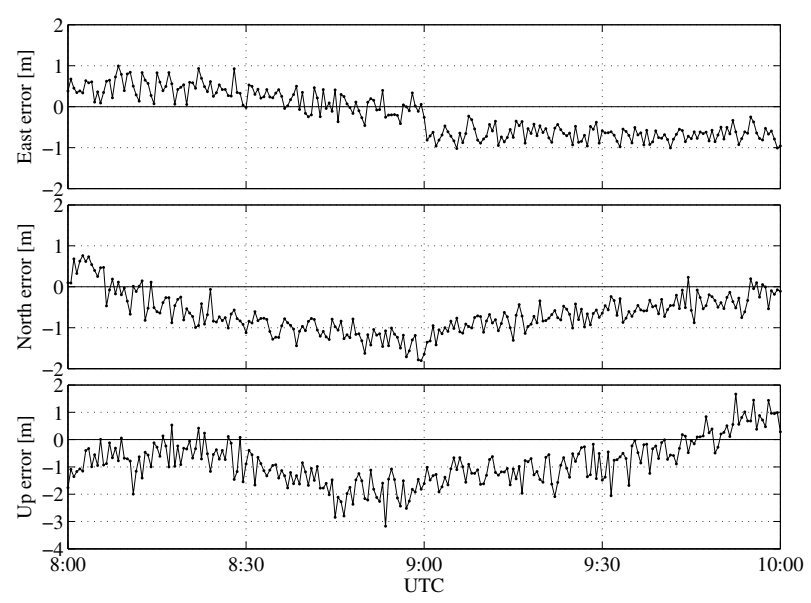

Fig. 9 Positioning errors (Single-Layer)

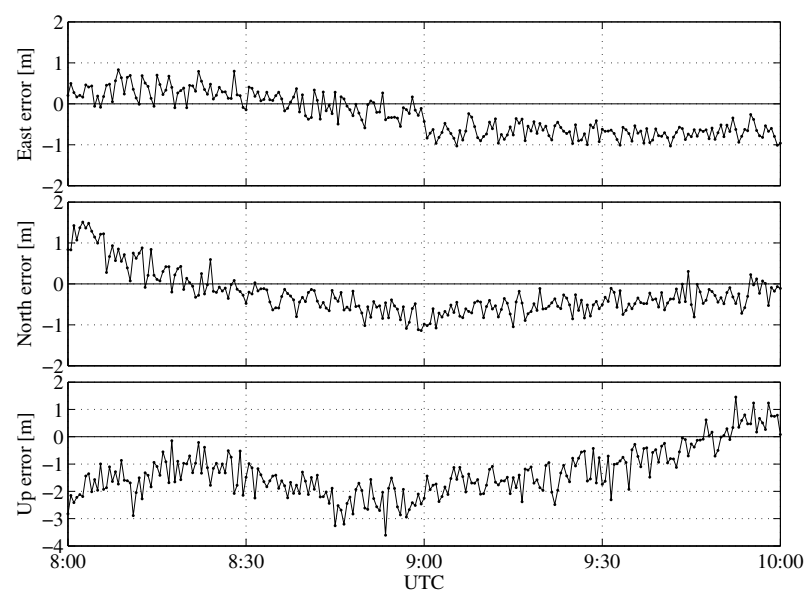

Fig. 10 Positioning errors (3-Layer)

\section{Conclusions}

In this paper, by using the GR models for GNSS observables at reference stations, we derived the method to estimate the ionospheric delays based on the multi-layer ionospheric model. We assumed the ionosphere has 3 spherical shells of different heights
Table 4 Position errors

\begin{tabular}{|c|c|r|r|r|}
\hline Method & Dir. & Bias[m] & STD $[\mathrm{m}]$ & RMS[m] \\
\hline \hline \multirow{4}{*}{ GIM } & East & -0.5426 & 0.5046 & 0.7402 \\
\cline { 2 - 5 } & North & -0.5643 & 0.4572 & 0.7257 \\
\cline { 2 - 5 } & Up & -2.0197 & 0.6775 & 2.1298 \\
\cline { 2 - 5 } & $2 \mathrm{D}$ & 0.7828 & 0.6809 & 1.0366 \\
\hline \hline \multirow{3}{*}{$\begin{array}{l}\text { Single- } \\
\text { Layer }\end{array}$} & East & -0.2020 & 0.5427 & 0.5780 \\
\cline { 2 - 5 } & North & -0.6654 & 0.4842 & 0.8223 \\
\cline { 2 - 5 } & Up & -0.8487 & 0.8328 & 1.1878 \\
\cline { 2 - 5 } Multi- & $2 \mathrm{D}$ & 0.6954 & 0.7273 & 1.0052 \\
\cline { 2 - 5 } Layer & East & -0.2811 & 0.4870 & 0.5615 \\
\cline { 2 - 5 } & Up & -0.2496 & 0.5267 & 0.5818 \\
\cline { 2 - 5 } & $2 \mathrm{D}$ & 0.3759 & 0.7173 & 0.8085 \\
\hline \hline
\end{tabular}

and successfully separated by using least squares methods with constraint conditions. We compared the positioning errors with GIM, single-layer model and multi-layer model. As a result, the positioning accuracy applied by the ionospheric correction based on the multi-layer model was improved in the horizontal plane.

\section{References}

[1] S. Sugimoto and Y. Kubo: GNSS regressive models and precise point positioning; Proc. of 36th ISCIE Int. Symp. on Stochastic Sytems Theory and Its Applications, pp. 159-164 (2004)

[2] S. Sugimoto and Y. Kubo: Carrier-phase-based precise point positioning - A novel approach based on GNSS regressive equations; Proc. GNSS 2004 Symposium, Paper No. 94 (2004)

[3] Y. Kubo, S. Kitao, S. Fujita and S. Sugimoto: A new RTK algorithm for carrier-phase-based precise point positioning based on GNSS regression models; Proc. ION GNSS 2005, pp. 1492-1499 (2005)

[4] S. Sugimoto, Y. Kubo and S. Fujita: Carrier-phasebased PPP with multiple antennas based on GR models; Proc. of 2007 ION National Technical Meeting, pp. 881-891 (2007)

[5] S. Sugimoto and Y. Kubo: Unified methods of point and relative positioning based on GNSS regression equations; Proc. ION-GNSS 2006, pp. 345-358 (2006)

[6] Y. Kubo, S. Fujita and S. Sugimoto: Unified positioning algorithms based on GNSS regression equations - Further results of point and relative positioning; Proc. 2007 National Technical Meeting, The Institute of Navigation, pp. 892-902 (2007)

[7] S. Sugimoto, Y. Kubo, S. Fujita, T. Imamura and T. Kazuno: Ionospheric estimation over Japan based on GNSS regressive models and GEONET data; Proc. ION GNSS 2007, pp. 2346-2356 (2007)

[8] S. Fujita, H. Yamamoto, T. Iura, Y. Kubo and S. Sugimoto: Modeling ionosphere VTEC over Japan based on GNSS regression models and GEONET; Int. J. of Innovative Computing, Information and Control, Vol. 6, No. 1, pp. 155-169 (2010)

[9] H. Akaike: A new look at the statistical model iden- 
tification; IEEE Trans. Automatic Control, Vol. 19, No. 6, pp. 716-723 (1974)

[10] G. Schwarz: Estimation the dimension of a model; Annals of Statistics, Vol. 6, pp. 461-464 (1978)

[11] S. Otsuki, M. Kamimura, M. Ohashi, Y. Kubo and S. Sugimoto: Local models for ionospheric VTEC estimation based on GR models and spherical cap harmonic analysis; Journal of Aeronautics, Astronautics and Aviation, Vol. 43, No. 1, pp. 1-7 (2011)

[12] G. Xu: GPS - Theory, Algorithms and Applications, Springer-Verlag (2003)

[13] B. W. Parkinson and J. J. Spilker Jr. (Eds.): Global Positioning System: Theory and Applications, Vol. I, II, AIAA (1997)

[14] S. Schaer, W. Gurtner and J. Feltens: IONEX: The IONosphere Map EXchange format version 1, Proceedings of the IGS AC Workshop (1998)

[15] R. H. Byrd, J. C. Gilbert and J. Nocedal: A trust region method based on interior point techniques for nonlinear programming; Mathematical Programming, Vol. 89, No. 1, pp. 149-185 (2000)

\section{Appendix}

\section{Appendix 1. Abbreviations}

The abbreviations used(written) in this paper are summarized as follows.

$\begin{array}{lll}\text { AIC } & : & \text { Akaike's Information Criterion } \\ \text { BIC } & : & \text { Baysian Information Criterion } \\ \text { GEONET } & : & \text { Gps Earth Observation NETwork } \\ \text { GIM } & : & \text { Global Ionosphere Maps } \\ \text { GNSS } & : & \text { Global Navigation Satellite System } \\ \text { GPS } & : & \text { Global Positioning System } \\ \text { GR } & : & \text { GNSS Regressive } \\ \text { GSI } & : & \text { Geospatial Information Authority } \\ & & \text { of Japan } \\ \text { IGS } & : & \text { International GNSS Service } \\ \text { IPP } & : & \text { Ionospheric Pierce Point } \\ \text { PPP } & : & \text { Precise Point Positioning } \\ \text { SCHA } & : & \text { Spherical Cap Harmonic Analysis } \\ \text { SHF } & : & \text { Spherical Harmonic Functions } \\ \text { SPP } & : & \text { Standard Point Positioning } \\ \text { TEC } & : & \text { Total Electron Content } \\ \text { UTC } & : & \text { Universal Time Coordinated } \\ \text { VTEC } & : & \text { Vertical Total Electron Content }\end{array}$

\section{Authors}

Masaharu OHAshi (Student-Member)

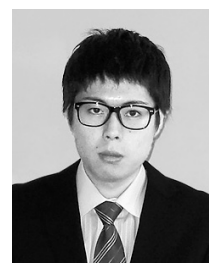

Masaharu Ohashi received the B.S. (2010) and M.S. (2012) degrees in Electrical and Electronic Engineering from Ritsumeikan University, Shiga, Japan in 2011. He is presently a Ph.D. student at graduate school of Science and Engineering, Ritsumeikan University.

Taisuke HATTORI

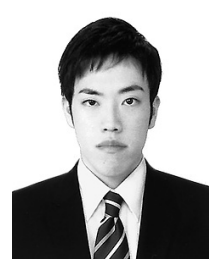

Taisuke Hattori received the B.S. degrees (2011) in Electrical and Electronic Engineering from Ritsumeikan University, Shiga, Japan in 2011. He is presently a M.S. student at graduate school of Science and Engineering, Ritsumeikan University.

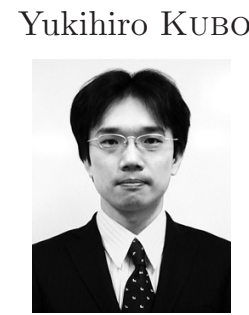

(Member)

Yukihiro Kubo received the B.S. (1997), M.S. (1999) and Ph.D. (2002) degrees in Electrical and electronic engineering from Ritsumeikan University, Shiga and Kyoto, Japan. He worked in the production section of GPS carnavigation systems at Mitsubishi Electric Corp., Sanda Works from 2002 to 2004. He joined Department of Electrical and Electronic Engineering of Ritsumeikan University in 2004, and he is presently an associate professor. His research interests include GPS/GNSS signal processing and INS/GNSS integration systems.

\section{Sueo Sugrmoto (Member)}

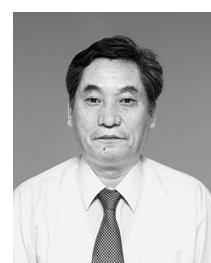

Sueo Sugimoto received the B.S. (1969) and M.S. (1971) degrees in mechanical engineering from the Kyoto Institute of Technology, Kyoto, Japan, and the Ph.D (1974) degree in electrical engineering system science from the Polytechnic Institute of New York (presently, Polytechnic Institute of NYU), New York. He is a professor, Department of Electrical and Electronic Engineering at Ritsumeikan University, Shiga and Kyoto, Japan. His research interests include stochastic systems, and statistical image-signal processing in various applications such as GPS/GNSS navigation. 